\title{
ALGORITHM AND ARCHITECTURE DESIGN FOR A LOW-COMPLEXITY ADAPTIVE EQUALIZER
}

\author{
Chun-Nan Chen, Kuan-Hung Chen, and Tzi-Dar Chiueh \\ Graduate Institute of Electronics Engineering and Department of Electrical Engineering, \\ National Taiwan University, Taipei, Taiwan 10617.
}

\begin{abstract}
As the need for multimedia communication continues to surge, consumers demand higher and higher transmission data rate. To circumvent the channel impairment caused by multipath fading, more and more receivers resort to adaptive equalizers. However, the complexity of time-domain adaptive equalizers can be too high for some specific applications. In this paper, a novel adaptive algorithm and its low-complexity architecture are proposed. This algorithm, called GSPT LMS algorithm, employs a new Grouped Signed Power-of-Two (GSPT) number representation. An adaptive equalizer using the proposed algorithm has been simulated and shown to be capable of equalization of 8PSK signals in several practical channels. Finally, the proposed adaptive equalizer and two other adaptive equalizers are implemented on FPGA. Simulation results show that the proposed architecture has the lowest complexity and saves about $50 \%$ to $70 \%$ of hardware.
\end{abstract}

\section{INTRODUCTION}

With the advent of modern communication technology and VLSI implementation, wireless communication services, such as mobile phones and indoor wireless networks have been advancing very rapidly. However, with the increase in transmission rate and signal constellation complexity, inter-symbol interference (ISI) caused by the multipath fading channel becomes more and more unbearable. To this end, equalizers have play an important role in removing ISI. Furthermore, adaptive equalizers have become popular since channels in mobile communication are constantly changing.

An adaptive equalizer is essentially a linear adaptive filter used to model the inverse transfer function of the channel. Two well-known adaptive algorithms are the least mean square (LMS) algorithm and the recursive least square (RLS) algorithm. Although the RLS algorithm has better convergence speed than the LMS algorithm, its complexity for hardware implementation can be very high. Actually, the LMS algorithm is widely adopted in hardware implementation because of its simplicity and robustness. Even so, power consumption is very crucial for many portable applications that use batteries. Therefore, new techniques for

This work was supported in part by MediaTek Inc. the algorithm and the implementation of equalizers are still needed to further reduce their complexity and power consumption.

An LMS adaptive equalizer can be divided into two parts: the feedforward filter and the updating unit. Multiplication operations are required in both parts. The canonical signed digit (CSD) representation, also known as signed power-oftwo (SPT), has been used for filter coefficients representation to reduce FIR filter complexity $[1,2,3]$. By employing such representations, multiplications required for the filtering process can be replaced by simple shift and add/subtract operations and the implementation complexity of the feedforward filter can be reduced. To reduce the implementation complexity of the updating part, several adaptive algorithms such as sign-sign, sign-error, sign-data, and log-log LMS algorithms have been proposed [4]. In addition, by utilizing these techniques, several multiplierless adaptive equalizers have been implemented $[5,6]$. One of the two equalizers, referred to Chen's scheme from now on, deserves detail description [6]. In Chen's scheme, the sign-data LMS algorithm is adopted for coefficient updating. The updated coefficient is then encoded by a modified radix -4 Booth algorithm. Suppose the coefficient wordlength is chosen as $t$ and the $t / 2$ encoded signed digits are numbered from 1 (LSB) to $t / 2$ (MSB). The encoded signed digits are then split into $t / 4$ groups and each group has two signed digits numbered with $x$ and $x-t / 4$. There is at most one nonzero signed digit allowed in each group and the larger signed digit is selected if it is nonzero, otherwise the smaller one is selected, resulting in only $t / 4$ signed digits in each tap.

In this paper, both the algorithm and the architecture of a new low-complexity adaptive equalizer are proposed. First, we propose a new number system, called grouped signed power-of-two (GSPT) number system. Next, an equalizer adaptation algorithm based on the GSPT number system is presented. The GSPT number system divides the signed digits of each number into several groups and allows at most one nonzero signed digit in one group. So, the complexity of the feedforward filter can be further reduced as compared to the traditional signed power-of-two number system. The adaptive algorithm operates like the sign-sign LMS algorithm with variable step-size controlled by a simple mechanism. The hardware complexity required to realize the 
adaptive algorithm is only slightly more than the sign-sign LMS algorithm.

The rest of the paper is organized as follows. In Section 2 , the GSPT number system and the GSPT LMS algorithm are presented. Then, simulation results of a GSPT LMS adaptive equalizer used in receiving 8PSK signals over several practical channels are presented in Section 3. In Section 4, FPGA implementation complexity of the proposed architecture, the LMS equalizer, and the architecture proposed in [6] are compared. Finally, Section 5 concludes this paper.

\section{GSPT LMS ALGORITHM}

The updating equation of the LMS algorithm can be described by the following equation

$$
W_{k+1}=W_{k}+\mu \cdot e_{k} \cdot x_{k},
$$

where $\mu$ represents the step-size; $W_{k}, e_{k}$, and $x_{k}$ represent the coefficient of the feedforward filter, the error signal, and the input signal at time $k$ respectively. While implementing a low-complexity adaptive equalizer, the LMS algorithm is usually simplified to the sign-error (sign-data) LMS algorithm by replacing $e_{k}\left(x_{k}\right)$ with simply the sign of $e_{k}\left(x_{k}\right)$. Actually, sign-sign LMS algorithm can be used to further reduce the implementation complexity by employing only the signs of $e_{k}$ and $x_{k}$ for updating. This fact implies that the updating in $W_{k}$ needs not be precise but only monotonic to guarantee the convergence. In light of this idea, we propose a new number representation system and an adaptation algorithm based on the new number system to reduce the complexity of both the feedforward filter and the updating unit.

\subsection{GSPT Number System ${ }_{i}$}

The proposed number system is called the Grouped Signed Power-of-Two (GSPT) number system. The idea is to partition the digits of a number into several groups and then represent each group by signed digits. Furthermore, each group can be represented by at most one nonzero signed digit ( 1 or -1 ). For example, if a 12-bit number is considered here and partitioned into 3 groups, then a number can be represented by the GSPT number system is shown as following:

$$
449=\underline{0010} \underline{0100} \underline{0001},
$$

where each group is marked by an underline and the signed digit ' -1 ' is represented by $\overrightarrow{1}$.

\subsection{Basic Updating Algorithm}

In the GSPT LMS adaptation algorithm, only the direction of the term, $\mu \cdot e_{k} \cdot x_{k}$, is considered in coefficient updating. The coefficient adaptation is given by

$$
W_{k+1}= \begin{cases}W_{k}+ & \text {, if } \mu \cdot e_{k} \cdot x_{k}>0 \\ W_{k} & \text {, if } \mu \cdot e_{k} \cdot x_{k}=0 \\ W_{k}- & \text {, if } \mu \cdot e_{k} \cdot x_{k}<0\end{cases}
$$

If the term, $\mu \cdot e_{k} \cdot x_{k}$, is positive (negative or zero), we increase (decrease or freeze) the feedforward filter coefficient $W_{k}$. An updating unit shown in Figure 1(a) is designed to perform the above updating algorithm and the updating operation is illustrated in Figure 1(b).

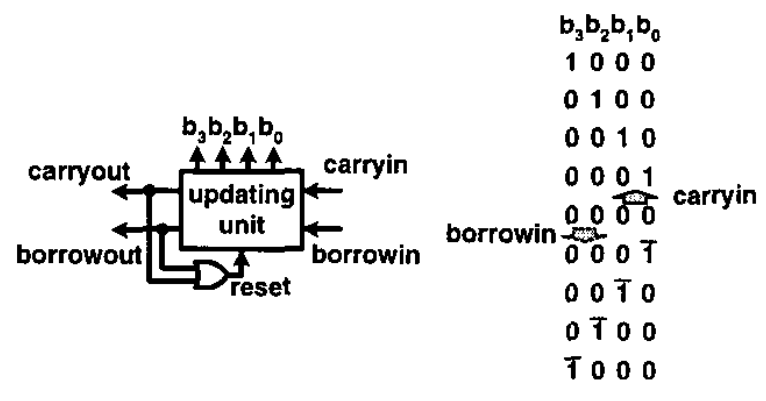

(a)

(b)

Figure 1: (a) Updating unit. (b) Illustration of the operation in an updating unit.

If a positive trigger signal (carryin) is received, $b_{3} b_{2} b_{1} b_{0}$ of this updating unit 'shifts up' to increase the value of this group. On the contrary, if a negative trigger signal (borrowin) is received, $b_{3} b_{2} b_{1} b_{0}$ of this updating unit 'shifts down' to decrease the value of this group. If $b_{3} b_{2} b_{1} b_{0}$ has been already at the topmost (bottom-most) limit $1000(\overline{1000})$ and a positive (negative) trigger signal is received, the output signal carryout (borrowout) is activated and $b_{3} b_{2} b_{1} b_{0}$ is reset to 0000 . Figure 2 shows the coefficient updater implemented by cascading three updating units in an equalizer with 12-bit coefficients. Notice that in Figure 2, some counters are introduced between two updating units. These counters act as buffers that accumulate the carryout or borrowout signals from the less-significant updating unit and trigger the carryin or borrowin signal to the more significant updating unit once a specified number of carryout or borrowout signals have been received. Basically, they are introduced to reduce the influence caused by fluctuations due to noise. Besides, these counters will decrease the convergence speed and a trade-off between the steady-state error and the convergence speed must be made carefully.

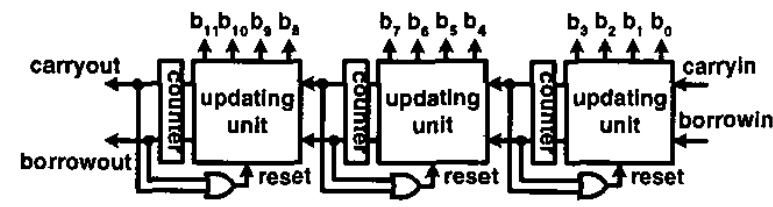

Figure 2: Coefficient updater. 


\subsection{GSPT-A LMS Algorithm}

It is well known that, for any adaptive algorithm, the smaller the updating step-size the lower the steady-state error. Even though the step-size is variable but not fixed in the GSPT LMS algorithm, we can decrease the step-size by increasing the wordlength of filter coefficients. To improve the steadystate error performance without increasing the complexity of the feedforward filter, we can increase the wordlength considered in updating but use only a more significant part of the coefficient in the feedforward filter. The concept is shown in Figure 3 and referred to as the GSPT-A LMS algorithm. However, the convergence speed will be slower than GSPT LMS algorithm due to a smaller step-size.

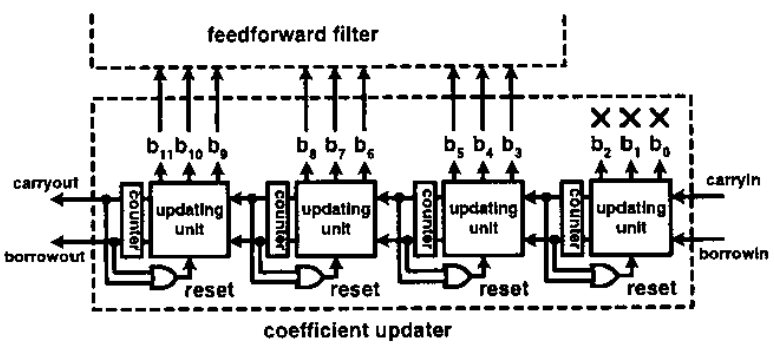

Figure 3: Coefficient updater for the GSPT-A LMS algorithm.

\section{SIMULATION RESULTS}

To verify the feasibility of the proposed adaptive algorithms in practical multipath fading channels, simulation of an adaptive equalizer in an 8PSK communication system is conducted. At the transmitter end, the bit stream is randomly generated and sent to an 8 PSK modulator. The modulated signal is then upsampled with over-sampling ratio of eight and the result is passed to a pulse shaping filter to generate the transmitter output. The transmitter output is then distorted by the multipath fading channel and AWGN. At the receiver end, the received signal is first passed through a pulse shaping filter, down-sampled, and sent to a T/4-spaced 41-tap adaptive equalizer. Finally, a decision block is used to decide the output symbol for calculating the symbol error rate. The GSM channel model [7] without Doppler effect is taken as the multipath fading channel for simulation.

Actually, there are several modifications to the LMS algorithm, such as sign-data, sign-error, and sign-sign LMS algorithms. However, these schemes converge much slower than the GSPT-A LMS, thus we compare only the GSPT LMS, GSPT-A LMS, and the conventional LMS in this section.

To begin with, the GSM channel without Doppler effect is selected as the multipath channel. The basic scheme of GSPT LMS algorithm is applied to the adaptive equalizer with different parameters. The group size, $g$, is chosen as
3 or 4 , while the upper bound of the counter, $c$, is chosen as 1 or 2 . The wordlength $w$ considered for updating is set to 12 . The simulation results on typical urban (TU) channel and hilly terrain (HT) channel are plotted in Figure 4. It is not surprising that GSPT LMS with $g=3$ has much better performance than $g=4$ while the performance with $c=2$ is superior to $c=1$ case regardless of the group size. Notice that the symbol error rate is very close for all schemes in low $E_{s} / N_{0}$ cases but has non-trivial difference in high $E_{s} / N_{0}$ cases. This phenomenon is caused by different coefficient precision in different schemes. The higher the precision, the better the error performance.
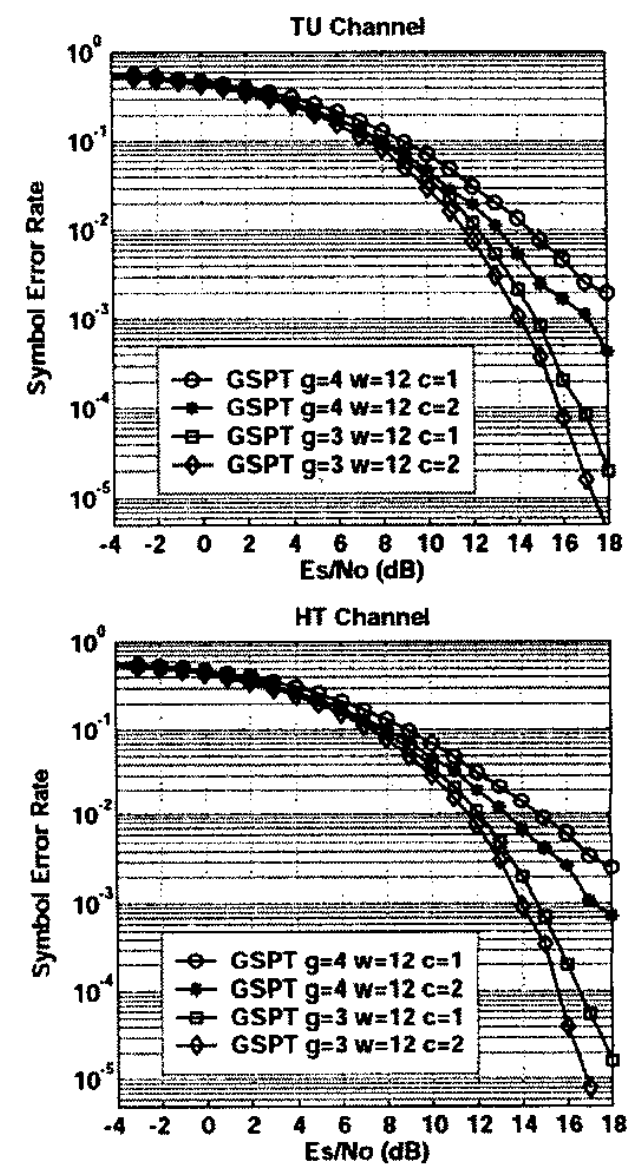

Figure 4: Symbol error rate versus $E_{s} / N_{0}$ on TU channel and HT channel using the GSPT LMS algorithm with different parameters.

Next, we compare the error performance between GSPT LMS, GSPT-A LMS, and LMS algorithm by simulations. The group size is chosen as 3 while the updating wordlength is set at 12 and 15 for GSPT and GSPT-A, respectively. The simulation results shown in Figure 5 indicate that GSPT-A is superior to GSPT on error performance as expected and is very close to the LMS algorithm. 

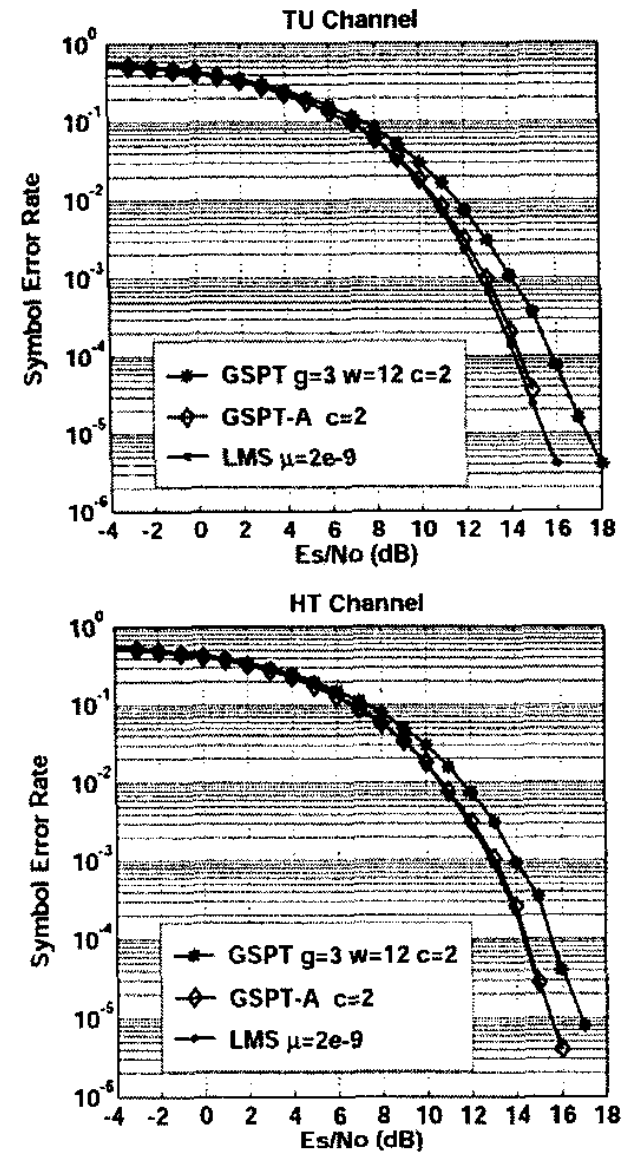

Figure 5: Symbol error rate versus $E_{s} / N_{0}$ on TU channel and HT channel: comparison between LMS, GSPT and GSPT-A.

\section{FPGA IMPLEMENTATION}

In this section, three adaptive equalizers based on the LMS algorithm, Chen's scheme, and the proposed GSPT-A LMS algorithm are implemented on FPGA to compare the implementation complexity. An 11-tap adaptive equalizer is adopted for FGPA implementation. Parameters of the GSPTA algorithm are chosen as $g=3, w=15$, and $c=1$. The coefficient wordlength of the feedforward filter is chosen as 12 for all three schemes.

Table 1 lists the number of logic element (LE) used for three different architectures. It is obvious that the proposed GSPT-A LMS algorithm can be implemented with much lower complexity, which is about $30 \%$ of the hardware resource required by the conventional LMS algorithm, or about $50 \%$ of that in Chen's scheme.
Table 1: Implementation complexity comparison between LMS, Chen's scheme, and GSPT-A.

\begin{tabular}{|c|c|c|c|c|c|c|}
\hline \multirow{2}{*}{} & \multicolumn{2}{|c|}{ LMS } & \multicolumn{2}{c|}{ Chen's scheme } & \multicolumn{2}{c|}{ GSPT-A } \\
\cline { 2 - 7 } & LE & $\%$ & LE & $\%$ & LE & $\%$ \\
\hline filter & 6645 & 100 & 7495 & 113 & 3172 & 48 \\
\hline update & 6908 & 100 & 688 & 10 & 953 & 14 \\
\hline total & 13533 & 100 & 8183 & 60 & 4125 & 30 \\
\hline
\end{tabular}

\section{SUMMARY}

In this paper, a low-complexity equalizer algorithm based on a new number system (GSPT) is proposed. Simulations for the GSPT algorithm and an improved scheme, GSPT-A, verify their advantages and feasibility. FPGA implementation results show that the GSPT-A has the lowest complexity, which is about $30 \%$ of that in the LMS equalizer or $50 \%$ of that in Chen's scheme.

\section{REFERENCES}

[1] Y. M. Hasan, L. J. Karem, M. Falkinburg, A. Helwig, and M. Ronning, "Canonic Signed Digit Chebyshev FIR Filter Design," IEEE Signal Processing Letters, vol. 8, pp. 167-169, June 2001.

[2] R. M. Hewlitt and E. S. Swartzlantler Jr., "Canonical Signed Digit Representation for FIR Digital Filters," IEEE Workshop on Signal Processing Systems, pp.416-426, 2000.

[3] Y. C. Lim, R. Yang, D. N. Li, and J. J. Song, "Signed Power-of-Two Term Allocation Scheme for the Design of Digital Filters," IEEE Trans. on Circuits and Systems II : Analog and Digital Signal Processing, vol. 46, issue 5, pp. 577-584, May 1999.

[4] S. S. Mahant-Shetti, S. Hosur, and A. Gatherer, "The Log-Log LMS Algorithm," in Proc. of IEEE Conf. on Acoustics, Speech, and Signal Processing, 1997, pp. 2357-2360.

[5] H. F. Chi, "A High-speed RSD Adaptive Filter Architecture with a Fast Carry-free SPT Converter," in Proc. IEEE ISCAS-99, 1999, pp. 187-190.

[6] C. L. Chen, K. Y. Khoo, and A. N. Wilson Jr., "A Simplified Signed Powers-of-two Conversion for Multiplierless Adaptive Filters," in Proc. IEEE ISCAS-96, 1996, pp. 364-367.

[7] P. Dent, G. E. Bottomley, and T. Croft, "Jakes Fading Model Revisited," Electronic Letters, vol. 29, no. 13, pp. 1162-1163, June 1993. 